\title{
Taquicardia paroxística supraventricular en lactantes. A propósito de un caso
}

\author{
FM. Ortiz Sanjuán ${ }^{\mathrm{a}}$, S. Pastor Bataller ${ }^{\mathrm{a}}$, C. Mas Lázaroa, \\ B. Tomás Aguirre ${ }^{b}$, A. Calabuig Fresquet ${ }^{c}$, I. Salort Jiménez ${ }^{c}$ \\ ${ }^{a}$ Residentes Medicina Familiar y Comunitaria. \\ ${ }^{b}$ Pediatra. Servicio de Pediatría. \\ 'Enfermería. Servicio de Urgencias. \\ Hospital Francesc Borja. Gandía, Valencia.
}

Rev Pediatr Aten Primaria. 2009; I 1:89-95

Francisco Miguel Ortiz Sanjuán, forsan@comv.es

\section{Resumen}

La taquicardia paroxistica supraventricular (TPSV) es la arritmia más frecuente en Pediatría y constituye una urgencia médica. Se presenta en un 0,1-0,4\% de la población pediátrica, la mayoría de ocasiones como hallazgo casual o palpitaciones, siendo bien tolerada y en ocasiones de resolución espontánea.

No obstante, suele requerir ingreso hospitalario preferentemente en una unidad de cuidados intensivos (UCI), ya que puede desencadenar arritmias malignas, insuficiencia cardiaca y miocardiopatía dilatada, sobre todo en lactantes. La evolución a muerte oscila del 1\% en pacientes con cardiopatía al 0,25\% en los pacientes sin cardiopatía asociada.

El tratamiento basado en la realización de maniobras vagales y la administración de adenosina suele ser efectivo aunque recientemente ha venido constatándose la escasa eficacia de las dosis bajas de ATP (50-100 $\mu \mathrm{g} / \mathrm{kg})$, imperando una revisión de los protocolos actuales.

En ocasiones, la primera asistencia es prestada en centros de Atención Primaria, incluso centros rurales con accesibilidad limitada a un centro hospitalario. A nuestro entender es básico que el médico de Atención Primaria conozca el manejo de esta patología y también que Enfermería sea adecuadamente entrenada, sobre todo en la obtención de vías venosas periféricas con el fin de prestar una adecuada asistencia al enfermo, más aún si no es posible organizar el traslado medicalizado del paciente en un lapso de tiempo razonable.

Es nuestra intención llamar la atención sobre dicha patología y con ayuda de un caso clínico centrar los aspectos básicos de su diagnóstico y manejo terapéutico, especialmente en lactantes pequeños.

Palabras clave: Taquicardia supraventricular, Adenosina, Pediatría. 


\section{Abstract}

Paroxistical supraventricular tachycardia is the most frequent arrhythmia in Pediatrics and it is a medical emergency. It happens in $0.1-0.4 \%$ of the pediatric population, mostly as an accidental finding or palpitations, it is well tolerated and sometimes with a spontaneous resolution.

Nevertheless, it frequently requires admission preferably in an intensive care unit $(U C I)$, since it can be followed by malignant arrhythmias, cardiac insufficiency and dilated myocardiopathy, especially in infants. The evolution to death goes from $1 \%$ in patients with cardiopathy to 0,25\% in patients without cardiopathy.

The treatment based on vagal stimulation manoeuvres and Adenosine (ATP) is usually successful, although recently has been communicated the low effectiveness of low doses of ATP (50-100 lg/ kg), making necessary a review of current protocols.

Occasionally, the first assistance is given at primary care centres, even at rural medical centres. So we think that it is basic that primary care doctors knew about this pathology and also that nurses must be trained in periferical venous access, especially if the patient cannot be moved to a reference centre in a brief time.

We wish to draw the attention to this condition and with the help of a clinical case to show the basics of its diagnosis and treatment, especially in infants.

Key words: Supraventricular tachycardia, Adenosine, Pediatrics.

\section{Introducción}

La taquicardia paroxística supraventricular (TPSV) es la arritmia más frecuente en Pediatría si excluimos las extrasístoles y constituye un motivo frecuente de consulta hospitalaria. Su incidencia en la población pediátrica podría estimarse en un $0,1-0,4 \%{ }^{1}$. Aproximadamente el $60 \%$ de los niños con taquicardia supraventricular desarrolla su primer episodio durante el primer año de vida².

Suele aparecer de forma súbita, algunas veces sin desencadenante claro, otras en contexto de procesos febriles, infecciones respiratorias, tras esfuerzos físicos, y postcirugía cardiovascular. Debido a la inmadurez del tejido de conducción cardiaco con presencia de vías accesorias y mayor propensión a desarrollar arritmias, puede presentarse en lactantes completamente sanos. En niños de mayor edad en ausencia de cardiopatía estructural suele asociarse a la práctica deportiva. También puede desencadenarla cualquier tipo de shock, anemia, ansiedad, insuficiencia cardiaca congestiva, enfermedades del miocardio, exceso de catecolaminas o tirotoxicosis.

Las frecuencias cardiacas diagnósticas de TPSV varían entre 130 y 300 latidos por minuto, en función de la edad del paciente y del mecanismo por el que se produce la taquicardia. En menos de un 10\% de los casos el intervalo QRS puede ser ancho a resultas de una conducción antidrómica'.

La TPSV tiene su origen por encima del Haz de His y en la mayoría de casos es de- 
bida a un mecanismo de reentrada en el nodo aurículoventricular (microreentrada) o bien a nivel aurículoventricular (macroreentrada). En niños menores de 12 años la taquicardia suele tener su origen en una vía o vías accesorias. En adolescentes suele ser más frecuente la taquicardia por reentrada intranodal.

En los lactantes, la forma de presentación más frecuente (como es nuestro caso) suele ser su hallazgo en una revisión médica de rutina $y$, en segundo lugar, la clínica inespecífica (vg. irritabilidad, mala coloración de piel y mucosas, rechazo del alimento). En los pacientes de mayor edad la clínica más frecuente son las palpitaciones. La sintomatología inespecífica en los más pequeños conlleva una mayor demora en el diagnóstico, por lo que estos presentan con mayor frecuencia insuficiencia cardiaca. El tratamiento basado en la realización de maniobras vagales y la administración de adenosina o trifosfato de adenosina (ATP) suele ser efectivo. La dosis inicial se establece en $50 \mu \mathrm{g} / \mathrm{kg}$, aumentando de 50 en $50 \mu \mathrm{g}$ hasta 300 $\mu \mathrm{g} / \mathrm{kg}$, aunque recientemente ha venido constatándose la escasa eficacia de las dosis bajas de adenosina (50-100 $\mu \mathrm{g} / \mathrm{kg}$ ).

\section{Caso clínico}

Recién nacido de 29 días de edad y $4,100 \mathrm{~kg}$ de peso llevado por sus padres al centro de salud para valoración por exantema facial. Al explorarlo su pediatra observa taquicardia y taquipnea, comprobando por pulsioximetría una frecuencia cardiaca de 255 ppm y Sat $\mathrm{O}_{2}: 97 \%$, por lo que lo remite al hospital. Hasta ese momento toleraba bien la alimentación y presentaba una buena ganancia ponderal.

El embarazo y parto transcurrieron con normalidad, siendo a término con Apgar $8 / 9$, un peso al nacimiento de $3.250 \mathrm{~g}$, talla de $51,5 \mathrm{~cm}$ y perímetro craneal de $31,8 \mathrm{~cm}$. No se constataron antecedentes familiares de interés.

A su llegada a urgencias presenta buen aspecto general con buen color, taquipnea y tiraje subcostal, una frecuencia cardiaca superior a 250 pulsaciones por minuto (ppm) con una Sat $\mathrm{O}_{2}$ de $97 \%$. A los pocos minutos presenta empeoramiento del estado general, frialdad y cianosis acras y palidez facial. El ECG (figura 1) presentaba morfología de taquicardia supraventricular con complejos QRS estrechos, ondas $P$ retrógradas y una frecuencia cardiaca de 288 ppm.

Se practican maniobras vagales (hielo en la cara) sin efecto, por lo que se administra 1 bolo intravenoso (IV) de $50 \mu \mathrm{g} / \mathrm{kg}$ de adenosina sin respuesta y 3 minutos después un segundo bolo de $100 \mu \mathrm{g} / \mathrm{kg}$, cediendo la taquicardia y revirtiendo a ritmo sinusal con una frecuencia de 168 


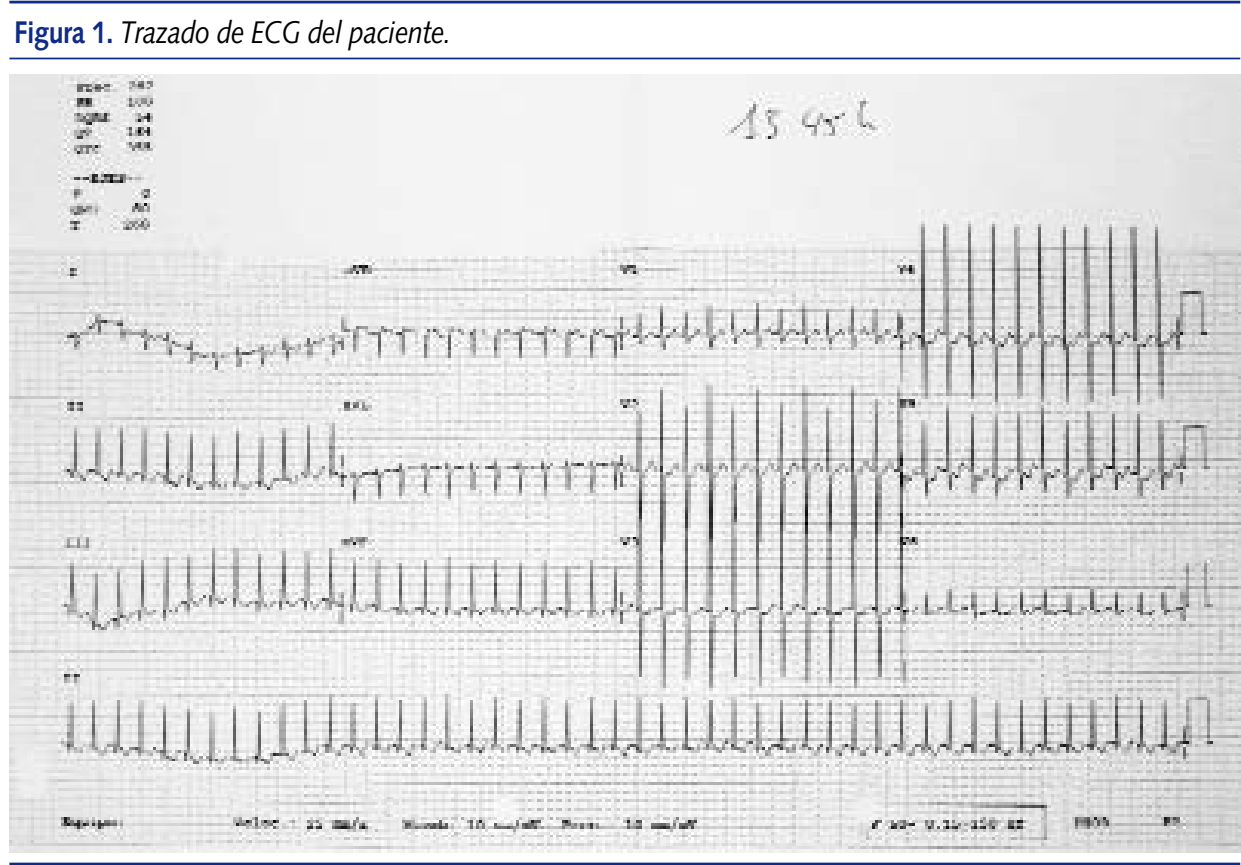

ppm y presentando mejoría de la coloración cutánea y del aspecto general.

Dado que en nuestro medio disponemos de ampollas de Adenocor ${ }^{\otimes}$ de $2 \mathrm{ml}$ con $6 \mathrm{mg}$ de fármaco, fue necesario realizar diluciones. En nuestro caso tomamos $0,5 \mathrm{ml}(1,5 \mathrm{mg})$ diluidos con 14,5 $\mathrm{ml}$ de suero fisiológico, consiguiendo una concentración de $100 \mu \mathrm{g} / \mathrm{ml}$. Se administra pues una dosis inicial de $2 \mathrm{ml}(50 \mu \mathrm{g} / \mathrm{kg})$ y ante la falta de respuesta una segunda dosis de $4 \mathrm{ml}(100 \mu \mathrm{g} / \mathrm{kg})$.

Tras la resolución de la taquicardia se procede a trasladar al paciente al hospital de referencia, a la Unidad de Cuidados Intensivos pediátrica (UCIP) para segui- miento y estudio. Durante las 24 horas siguientes, presentó un nuevo episodio de taquicardia supraventricular también resuelto con adenosina, manteniéndose posteriormente asintomático. Se informó de ecocardiografía normal. Hasta la fecha no ha presentado recurrencia alguna, manteniendo tratamiento profiláctico con propanolol.

\section{Discusión}

La taquicardia supraventricular, bien tolerada por la mayoría de los niños sanos, suele requerir ingreso hospitalario preferentemente en una UCIP, ya que puede desencadenar arritmias malignas, 
insuficiencia cardiaca y miocardiopatía dilatada, sobre todo en lactantes. Los pacientes menores de 3 meses o con inmediata recurrencia de la taquicardia tienen una alta probabilidad de recurrencia y complicaciones, por lo que requieren indefectiblemente ingreso hospitalario ${ }^{3}$. Según series, un 30-40\% de los pacientes menores de un año pueden presentar recurrencias². La maduración del sistema de conducción cardiaco suele producirse durante el primer año de vida, por lo que aproximadamente el $30 \%$ de los pacientes pierde la susceptibilidad para generar taquicardias alrededor de esta edad ${ }^{4}$. La evolución a muerte en esta patología oscila del $1 \%$ en pacientes con cardiopatía al $0,25 \%$ en los pacientes sin cardiopatía asociada ${ }^{1}$.

La persistencia en el tiempo de frecuencias cardiacas excesivamente elevadas, además de insuficiencia cardiaca congestiva, puede dar origen también a la denominada "taquimiocardiopatía" o miocardiopatía inducida por taquicardia ${ }^{5}$. Si bien la taquicardia puede permanecer asintomática mucho tiempo antes de claudicar el ventrículo izquierdo, es importante realizar un diagnóstico acertado, pues ésta es una de las pocas causas de miocardiopatía dilatada reversible, tanto por la normalización de la fracción de acortamiento como por la reducción del diámetro diastóli- co del ventrículo izquierdo ${ }^{6}$. Salerno et al. ${ }^{7}$ han identificado la edad inferior a los 3 años como factor de buen pronóstico para la taquimiocardiopatía inducida por taquicardia auricular ectópica, así como una frecuencia de resoluciones espontáneas del $76 \%$ en este grupo de edad, recomendando el tratamiento médico en los menores de 3 años y decantándose por la ablación mediante radiofrecuencia en los mayores.

En el estudio de estos pacientes es básica una ecocardiografía cardiaca para descartar cardiopatía estructural, además de valorar la funcionalidad.

El tratamiento inicial se basa en la práctica de maniobras vagales (en lactantes colocar una bolsa de hielo sobre la facies del niño, masaje del seno carotídeo en niños mayores) y cuando estas no son efectivas, la adenosina o trifosfato de adenosina (ATP) se ha convertido en el fármaco de elección, aunque si éste falla también han sido utilizados digoxina, verapamilo (contraindicado en menores de 1 año), propanolol y amiodarona (recalcar que esta última puede provocar patología tiroidea) e incluso la cardioversión eléctrica sincronizada. Recordar que ante la presencia de síndrome de Wolf-ParkinsonWhite está vetada la administración de fármacos bloqueadores del nodo aurículoventricular (ya que desviaríamos la tota- 
lidad de los impulsos auriculares a través de la vía accesoria).

Los protocolos de tratamiento recomiendan la administración seriada de adenosina hasta conseguir el cese de la taquicardia. La dosis inicial se establecía en $50 \mu \mathrm{g} / \mathrm{kg}$, aumentando de 50 en 50 $\mu \mathrm{g}$ hasta $300 \mu \mathrm{g} / \mathrm{kg}$. Recientemente ha venido constatándose la escasa eficacia de dichas dosis iniciales de adenosina (50$100 \mu \mathrm{g} / \mathrm{kg})^{8,9}$. En la serie de Dixon et al. ${ }^{10}$ la dosis de $50 \mu \mathrm{g} / \mathrm{kg}$ fue efectiva solo en el $9 \%$ de los pacientes y la dosis media efectiva de adenosina fue de $200 \mu \mathrm{g} / \mathrm{kg}$. De hecho, en la práctica clínica suele ser habitual emplear dosis de ATP de entre 300, 600 e incluso $900 \mu \mathrm{g} / \mathrm{kg} /$ dosis $^{1}$. Creemos pues necesaria la revisión de los protocolos actuales.

Una vez controlado el episodio agudo, el tratamiento ideal sería la ablación por radiofrecuencia de la vía o vías accesorias arritmogénicas. Dado que en numerosas ocasiones la propia maduración del sistema de conducción conlleva el cese de las taquicardias, la indicación de la ablación sería la imposibilidad de controlar los episodios con tratamiento médico.

\section{Conclusión}

La TPSV en la infancia constituye una urgencia médica ante la que debe actuarse con la máxima premura y eficacia siguiendo los protocolos de actuación vigentes, no siendo en ningún caso conveniente dilatar el tratamiento. Se impone pues el dominio del manejo prehospitalario de la misma, en el que no es necesario el diagnóstico preciso de la variante taquicárdica para iniciar tratamiento. Será básico clasificar al paciente como estable o inestable clínicamente, pues estos últimos requerirán cardioversión eléctrica o farmacológica.

Los protocolos de tratamiento recomiendan la administración seriada de ATP hasta conseguir el cese de la taquicardia, aunque quizá sería necesaria una revisión de los mismos con objeto de aumentar las dosis iniciales a $150 \mu \mathrm{g} / \mathrm{kg}$.

Consideramos de suma importancia el adecuado entrenamiento del médico de Atención Primaria así como del colectivo de enfermería en el manejo de la taquicardia supraventricular infantil. 


\section{Bibliografía}

1. Balaguer Gargallo M, Jordán García I, Caritg Bosch J, Cambra Lasaosa FJ, Prada Hermogenes F, Palomaque Rico A. Taquicardia paroxística supraventricular en el niño y el lactante. An Pediatr (Barc). 2007;67:133-8.

2. Tortoriello TA, Snyder CS, Smith EO, Fenrich AL Jr., Friedman RA, Kertesz NJ. Frequency of recurrence among infants with supraventricular tachycardia and comparison of recurrence rates among those with and without preexcitation and among those with and without response to digoxine and/or propanolol therapy. Am J Cardiol. 2003;92:1045-9.

3. Pudpud AA, Linares MY, Greenberg B. Is hospitalization necessary for treatment of SVT? Predictive variables for recurrence and negative outcome. Am J Emerg Med. 1999;17:512-6.

4. Etheridge S, Judd V. Supraventricular Tachycardia in infancy. Arch Pediatr Adolesc Med. 1999;153:267-71.

5. Rosés i Moguer F, Albert Brotons DC, Ferrer
Menduiña $Q$, Gran Ipiña F, Escobar Díaz MC, Moya Mitjans A. Miocardiopatía secundaria a taquicardia auricular ectópica. An Pediatr (Barc). 2006; 65:263-5.

6. Sánchez Fernández-Bernal C, Benito Bartolomé F. Reversibilidad de la miocardiopatía inducida por taquicardia supraventricular incesante en niños tras ablación con radiofrecuencia. Rev Esp Cardiol. 1997;50:643-9.

7. Salerno J, Kertesz NJ, Friedman R, Fenrich AL. Clinical course of atrial ectopic tachycardia is age-dependent: results and treatment in children $<3$ or $>$ or $=3$ years of age. J Am Coll Cardiol. 2004;43: 438-44.

8. Gandhi A, Uzun O. Adenosine dosing in supraventricular tachycardia: time for change. Arch Dis Child. 2006;91:373-5.

9. Rosenthal E. Pitfalls in the use of adenosine. Arch Dis Child. 2006;91:451-3.

10. Dixon J, Foster K, Wyllie J, Wren C. Guidelines and adenosine doping in supraventricular tachycardia. Arch Dis Child. 2005;90: 1190-1. 